\title{
Carotid sinus nerve section abolishes NMDA evoked respiratory effects in anaesthetised rats
}

\author{
Katarzyna Kaczyńska*, Małgorzata Szereda-Przestaszewska \\ ${ }^{a}$ Laboratory of Respiratory Reflexes, Polish Academy of Sciences Medical Research Centre, 5 Pawinskiego St., 02-106 Warsaw, Poland
}

Accepted 20 July 2004

\begin{abstract}
Respiratory effects of NMDA injection into the right atrium were investigated in 11 urethane-chloralose anaesthetised and spontaneously breathing rats. The animals were initially vagotomised and six of them were subdued to the subsequent carotid sinus nerve section, and the other five were treated by NMDA antagonist. Bolus injection of NMDA $(27 \mu \mathrm{mol} / \mathrm{kg})$ induced the depression of ventilation in all rats, due to the decrease in tidal volume from a baseline of $2.98 \pm 0.4$ to $2.63 \pm 0.3 \mathrm{ml}(P<$ $0.01)$, and slowing down of the respiratory rate from a baseline of $56 \pm 2.6$ to $27 \pm 2.0$ breaths $\min ^{-1}(P<0.0001)$. Section of the carotid sinus nerves (CSNs) precluded the respiratory depression. Prolongation of the expiratory time was reduced by this neurotomy from $5.07 \pm 2.6$ to $1.04 \pm 0.03(P<0.05)$. In five rats the blockade of NMDA receptors with the selective antagonist (AP-7) was likewise efficient in eliminating the post-NMDA respiratory response. NMDA increased mean arterial blood pressure and this rise occurred beyond the afferentation from the carotid bodies and the blockade of NMDA receptors. Results of this study indicate that inhibition of the respiratory drive evoked by NMDA administered via the peripheral circulation requires intact carotid bodies and activation of NMDA receptors.
\end{abstract}

(c) 2004 Elsevier B.V. All rights reserved.

Keywords: Control of breathing; Central NMDA receptors; Mammals; Rat; NMDA

\section{Introduction}

Glutamic and aspartic acids are generally believed to excite neural activity of both inspiratory and expiratory neurones, which is mediated, among others, via $\mathrm{N}$-methyl-D-aspartate receptors, subtype of the glutamate receptors (McCrimmon et al., 1995). Activa-

\footnotetext{
* Corresponding author. Tel.: +48 22608 6522; fax: +4822668 5532 .

E-mail address: kkacz@cmdik.pan.pl (K. Kaczyńska).
}

tion of central NMDA receptors by microinjection of their agonist: $N$-methyl-D-aspartic acid (NMDA) to the respiratory-related neurones of the nucleus tractus solitarii was described to evoke apnoeas and prolongation of expiration in the rat (Bonham et al., 1993; Berger et al., 1995). NMDA receptors are expressed in autonomic and sensory ganglia (Shigemoto et al., 1992) and in all regions of the lungs and airways in rats (Dickman et al., 2004). Therefore they may contribute to the peripheral reflex control of breathing. This problem received less of the attention. Recently we have provided 
evidence that excitation of the peripheral NMDA receptors in rats provokes the depression of breathing and the effect occurs beyond the lung vagal pathway (Kaczyńska and Szereda-Przestaszewska, 2004). Further, although NMDA receptors are densely distributed within the nodose ganglion (Aicher et al., 1999), supranodose vagotomy had little, if any, effect on the respiratory response induced by an intravenous NMDA challenge (Kaczyńska and Szereda-Przestaszewska, 2004).

The present study started with the hypothesis that the respiratory depression produced by the intraatrial NMDA challenge is mediated by the carotid body input to the medullary centres. This hypothesis has been tested by measuring the ventilatory effects of NMDA evoked in initially vagotomised rats and subsequent to the section of the carotid sinus nerves. To specify that the respiratory effects are due to action on peripheral NMDA receptors, we have examined whether the selective NMDA antagonist AP-7 blocks the responses.

\section{Methods}

Eleven adult Wistar rats (250-300 g body weight) were anaesthetised with an intraperitoneal (i.p.) injection of $600 \mathrm{mg} / \mathrm{kg}$ urethane (Sigma) and $120 \mathrm{mg} / \mathrm{kg}$ alpha-chloralose (Fluka AG). They were placed supine and breathed spontaneously room air. The trachea was exposed in the neck, sectioned below the larynx and the cannula inserted into the caudal end was connected to a pneumotachograph. Catheters were put into the femoral vein and into the right atrium (via an external jugular vein) for drug administration and to the femoral artery to monitor blood pressure. The midcervical segments of the vagi were cleared from adjacent tissue, dissected and initially sectioned. In six of eleven rats the carotid region on both sides was dissected under an operating microscope and carotid sinus nerves (CSNs) were prepared for section later in the experiment. Ethical approval for the experimental protocol used in the present study was obtained from the local animal care committee. All animal procedures were in accordance with the NIH Guide for the Care and Use of Laboratory Animals.

Tidal volume signals were recorded from the pneumotachograph (Electrospirometer C 56, Mercury) attached to the tracheal cannula. Arterial blood pressure was measured with BP-2 blood pressure moni- tor (Columbus Instruments) and mean arterial pressure was calculated (MAP). End-tidal $\mathrm{CO}_{2}$ concentration was measured with a capnograph (Engstrom Eliza Plus, Gambro). Electromyogram of the costal diaphragm was recorded with bipolar electrodes, amplified (NL 104, Digitimer), filtered and measured with model AS 101 (Asbit) leaky integrator (time constant $=100 \mathrm{~ms}$ ). The recordings were registered on an Omnilight 8 M 36 apparatus (Honeywell). Rectal temperature was maintained at $38^{\circ} \mathrm{C}$ with a heating pad.

$\mathrm{N}$-methyl-D-aspartic acid ([R]-2[methylamino]succinic acid, Sigma) at a dose of $27 \mu \mathrm{mol} / \mathrm{kg}$ dissolved in $0.9 \%$ saline was injected as a bolus to the right atrium. The dose of NMDA used in our study was derived from the dose-response relationship experiments, strictly reported elsewhere (Kaczyńska and SzeredaPrzestaszewska, 2004). The dose of $27 \mu \mathrm{mol} / \mathrm{kg}$ was found to be the most effective one to evoke the depression of respiratory variables. DL-2-amino-7phosphonoheptanoic acid (AP-7, Sigma) at a dose of $200 \mu \mathrm{g} / \mathrm{kg}$ (Sitniewska et al., 2003) was dissolved in $0.2 \mathrm{ml}$ aliquots of physiological saline injected intravenously. A flash of $0.2 \mathrm{ml}$ saline via a catheter placed in the right femoral vein or right atrium followed all drug administrations. Test i.v. or right atrium injections of $0.2 \mathrm{ml}$ aliquots of physiological saline showed no volume effects.

The respiratory effects evoked by an intraatrial NMDA challenge were recorded in 11 initially vagotomised rats then divided into two groups: (1) subsequently treated by section of the carotid sinus nerves $(n=6)$ and (2) by administration of NMDA receptors' blocker: AP-7 $(n=5)$.

Each individual value of the ventilatory parameters studied $\left(\mathrm{V}_{\mathrm{T}}, \mathrm{V}_{\mathrm{E}}\right.$, and $\left.\mathrm{f}\right)$ was taken as an average over five consecutive breaths. The ventilatory parameters were assessed prior to NMDA injection, during the early post-NMDA phase (immediately after the end of NMDA injection) and at 30 and $60 \mathrm{~s}$ after the challenge. The expiratory time $\left(T_{\mathrm{E}}\right)$ was determined from the record of integrated diaphragm activity. Prolongation of $T_{\mathrm{E}}$ was measured as the ratio of maximal $T_{\mathrm{E}}$ at $30 \mathrm{~s}$ after NMDA challenge ( $\left.T_{\text {ENMDA }}\right)$ to control expiration $\left(T_{\text {Econtrol }}\right)$. Ventilatory responses were assessed by comparing the mean of five consecutive breaths during the period of breathing following NMDA injection to the mean of five preceding breaths (control $=$ pre-challenge values) and are expressed as abso- 
lute changes. All experimental data were analysed by repeated measures two-way ANOVA with time (prechallenge, early post-NMDA phase, 30 and $60 \mathrm{~s}$ after the challenge) and innervation status (vagi cut and CSNs cut) as repeated measures factors. Differences between individual time points and experimental situations were evaluated by planned contrast analysis. Prolongation of $T_{\mathrm{E}}$ was analysed by Wilcoxon paired test or one-way ANOVA with innervation status as repeated measures factor. In all cases, a $P<0.05$ was considered significant. All results shown are means \pm S.E.M.

\section{Results}

Since it has been shown that the cardiorespiratory responses evoked by an intraatrial NMDA injection do not require vagal reflexes (Kaczyńska and SzeredaPrzestaszewska, 2004), therefore in the present study, the animals of the control groups were subdue to the initial bilateral midcervical vagotomy.

Right atrial injection of NMDA in the vagotomised rats produced uniform respiratory effects, comprising slow breathing of decreased tidal volume, which resulted in the decline in minute ventilation. There were no respiratory effects when similar volumes of the solvent were administered. Mean changes in the respiratory variables induced by NMDA administration are shown in Fig. 1. The tidal volume diminished in all animals (ANOVA, $P=0.0018$ ). Maximum significant lowest value of tidal volume was observed at the early phase and at $30 \mathrm{~s}$ after NMDA injection. Intraatrial injection of NMDA evoked a decrease in respiratory rate (ANOVA, $P=0.000002$ ), which lagged behind its effects on tidal volume. The mean decline in the respiratory rate achieved its significant maximum at 30 and $60 \mathrm{~s}$, compared to pre-drug control (Fig. 1). Minute ventilation was affected by NMDA (ANOVA, $P=0.000009)$ and reached the significant fall from the respective control values at 30 and $60 \mathrm{~s}$ (Table 1).

To determine whether the sinus nerves contribute to mediating the response to the right atrial NMDA injection, the drug was given in six vagotomised rats prior to and after CSNs section. Fig. 2 shows the respiratory response to the right atrial injection of NMDA in vagotomised (A) and CSNs-cut rat (B). The depression of respiration in initially vagotomised rats was completely
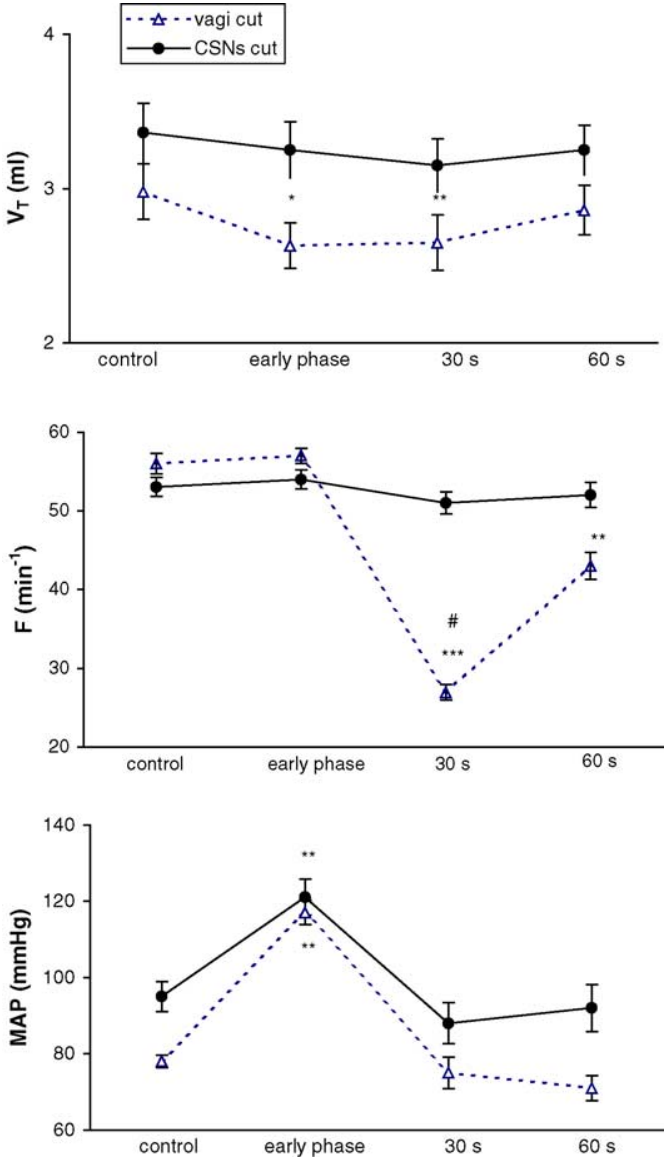

Fig. 1. Effects of NMDA on tidal volume, frequency of breathing, and mean arterial blood pressure in bilaterally vagotomised and CSNs sectioned rats. All values are mean \pm S.E.M., $n=6,{ }^{* * *} P<$ $0.001,{ }^{* *} P<0.01,{ }^{*} P<0.05$, vs. the respective pre-NMDA values, \#P $<0.05$ vs. the corresponding pre-carotid neurotomy value (two-way ANOVA followed by planned contrasts' analysis).

abolished by the subsequent section of the carotid sinus nerves.

Section of the CSNs eliminated the mean prolongation of $T_{\mathrm{E}}$ from $5.07 \pm 2.6$ in vagotomised to $1.04 \pm$ 0.03 in CSNs cut animals $(P<0.05)$.

Respiratory effects of NMDA were accompanied by an immediate rise in the mean arterial blood pressure in both neural states. The blood pressure reverted to the baseline level within 30 or $60 \mathrm{~s}$ (Fig. 1).

Use of the AP-7 permitted to verify the specifity of the NMDA bolus on NMDA receptors. As can be noted, pretreatment with AP-7 cancelled out the respiratory response to NMDA. There was no significant 
Table 1

Changes in minute ventilation $\left(\mathrm{V}_{\mathrm{E}}\right)$ after NMDA challenge into right atrium.

\begin{tabular}{|c|c|c|c|c|c|}
\hline \multirow[t]{3}{*}{ Innervation status } & \multirow[t]{3}{*}{$n$} & \multicolumn{4}{|c|}{$\mathrm{V}_{\mathrm{E}}\left(\mathrm{ml} \min ^{-1}\right)$} \\
\hline & & \multirow[t]{2}{*}{ Baseline } & \multicolumn{3}{|c|}{ After NMDA } \\
\hline & & & Early phase & $30 \mathrm{~s}$ & $60 \mathrm{~s}$ \\
\hline Vagi cut & 6 & $173 \pm 24$ & $153 \pm 21$ & $74 \pm 10^{* * *}$ & $128 \pm 19^{* *}$ \\
\hline CSNs cut & 6 & $177 \pm 17$ & $176 \pm 18$ & $158 \pm 16^{\#}$ & $172 \pm 20$ \\
\hline
\end{tabular}

All values are means \pm S.E.M.

$* P<0.05$.

** $P<0.01$ vs. the respective pre-NMDA value.

\# $P<0.001$ vs. the corresponding pre-CSNs cut value. Two-way ANOVA followed by planned contrasts' analysis.

change neither in tidal volume nor in the frequency of breathing when NMDA was administered after previous AP-7 application (Fig. 3). The prolongation of $T_{\mathrm{E}}$ induced beforehand by NMDA challenge attained 1.81 \pm 0.14 and was effectively precluded to $1.08 \pm 0.03$ $(P<0.01)$ by the antagonist. AP-7 did not abolish the rise in arterial blood pressure evoked by NMDA administration (Fig. 3). Hence, these data indicate that

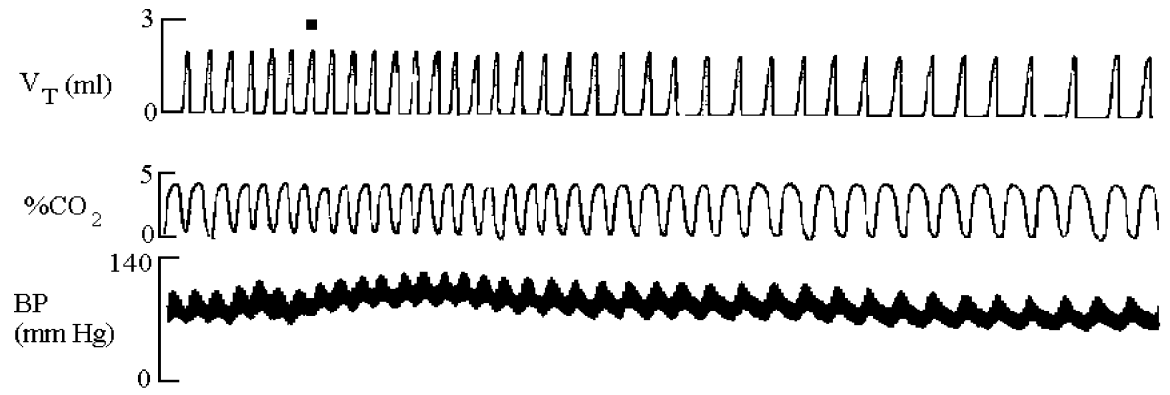

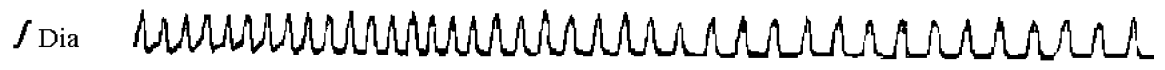

(A) vagi cut

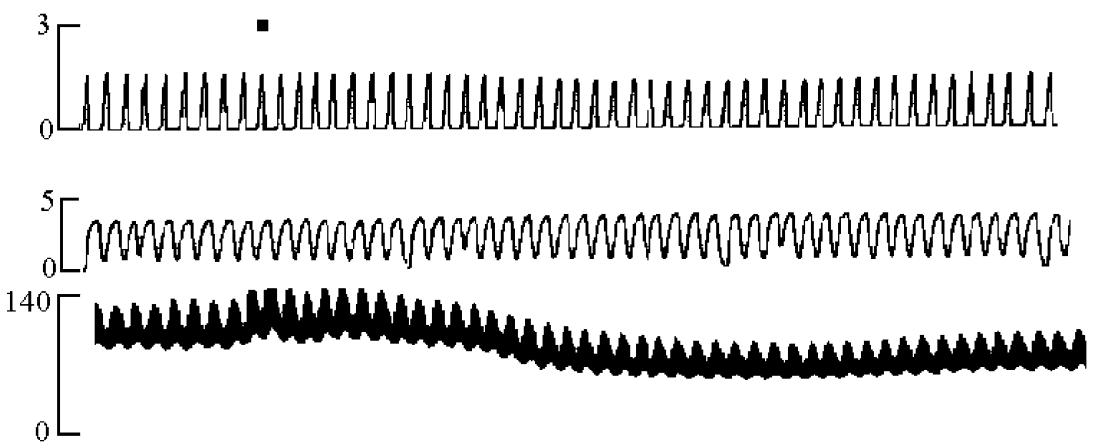

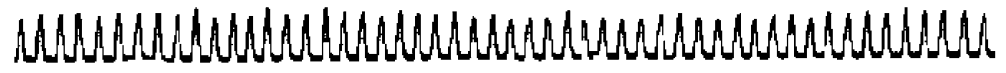

(B)

vagi + CSNs cut

Fig. 2. Respiratory response to intraatrial injection of NMDA in midcervically vagotomised rat (A). Injection marked by a dot above the upper record. Note slowing down of breathing with lowered tidal volume and an increase in blood pressure. Following CNSs neurotomy (B), the respiratory response to NMDA is absent. Apparent rise in blood pressure is followed by the depressive effect. $\mathrm{V}_{\mathrm{T}}$, tidal volume; $\% \mathrm{CO}_{2}$, end-tidal $\mathrm{CO}_{2}$; BP, arterial blood pressure; $\int \mathrm{Dia}$, integrated electromyogram of the diaphragm. 

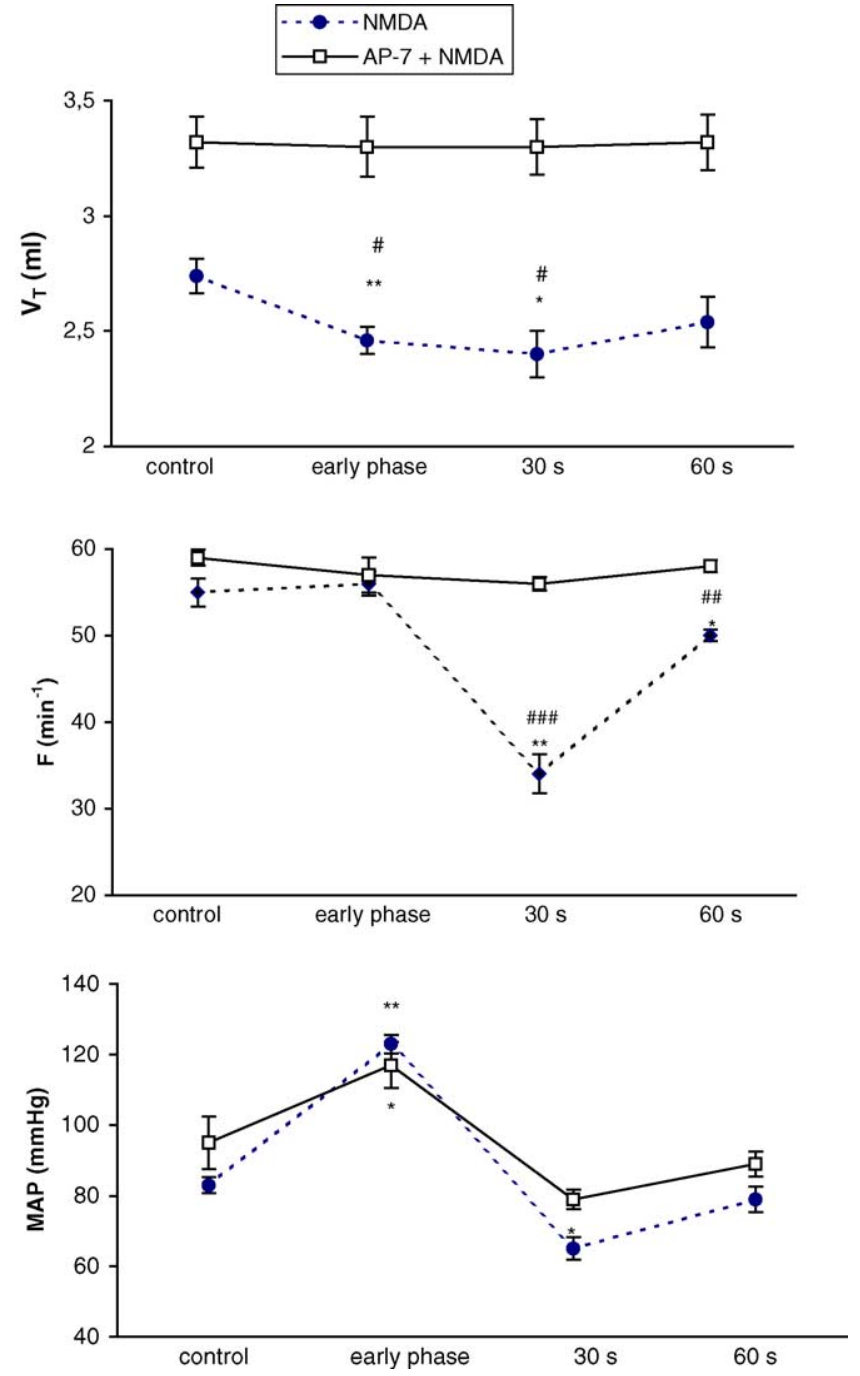

Fig. 3. Effects of AP-7 blockade on tidal volume, respiratory rate and blood pressure changes induced by NMDA in bilaterally vagotomised rats. (Mean \pm S.E.M., $n=5){ }^{* *} P<0.01,{ }^{*} P<0.05$ vs. the respective pre-NMDA value, ${ }^{\# \# \#} P<0.001,{ }^{\# \#} P<0.01,{ }^{\#} P=0.05$ vs. the corresponding value prior to blockade with AP-7 (two-way ANOVA followed by planned contrasts' analysis).

the blockade of NMDA receptors does not counteract the pressor effect of NMDA.

\section{Discussion}

The present study confirmed our earlier findings that intraatrial administration of NMDA in midcervically vagotomised rats evoked depression of respiration, affecting both constituents of the breathing pattern
(Kaczyńska and Szereda-Przestaszewska, 2004). Therefore the current experiments focused on whether the NMDA-induced respiratory response after removal of the sensory input from the lungs, relies on the chemoafferent nerves. The results obtained with AP-7 selective blocker showed that the depressive effect of NMDA on respiration in our experimental setup is mediated by an activation of the NMDA-ergic receptors. These latter may affect the respiratory system at several sites. The mediation via the supranodose vagal 
feedback was beforehand excluded (Kaczyńska and Szereda-Przestaszewska, 2004), and excitation of the central receptors seemed questionable, since NMDA is considered to have a low, if any, permeability through the blood-brain barrier. The presumption of peripheral site of NMDA action is consistent with the fact that, AP-7 which effectively antagonised respiratory response observed in our study, acts peripherally due to its limited transport across blood-brain barrier (Lodge and Danysz, 2000).

This is the first report comparing the respiratory response to NMDA administered via the peripheral circulation in midcervically vagotomised and subsequently bilaterally chemodenervated rats.

The major result of this study is that section of the carotid sinus nerves abolished the respiratory response to the right atrial injection of NMDA in our rats. The input from the aortic chemoreceptors was left intact and this shows that it does not contribute much to the respiratory effects of NMDA, similarly as to other ventilatory responses (Sapru and Krieger, 1977). The pattern of the respiratory response observed by us, is not characteristic for excitation of the peripheral chemoreceptors, which brings about an increased inspiratory volume and the rate of breathing. Yet, it follows slow and long-lasting effects of NMDA receptors' activation (McCrimmon et al., 1995). Our results imply, that injection of NMDA has an inhibitory effect on carotid chemoreceptors, resulting in a reduction in chemodrive and the depression of breathing. This is reinforced by the finding, that intravenous application of MK801 , an antagonist to the function of the NMDA receptors, increases ventilation (Harris and Milsom, 2001). Nevertheless, the mechanism underlying the action of NMDA on carotid chemoreceptors is far from being clarified. We have not found any reports concerning the expression of NMDA receptors in the rat carotid bodies, which would support our results. It is of note, however, that aspartate-ir neurones are contained in the petrosal ganglion of the rat (Okada and Miura, 1992). Among multiple neurotransmitters, which coexist in the carotid bodies and take part in the chemotransmission, high concentration of L-glutamate abounds but is not being released from the glomus cells (Torrealba et al., 1996). Analysis of the published data does provide evidence that glutamate is released from the primary afferent neurones of the peripheral chemoreceptors relaying to the nucleus tractus solitarii (Lewis et al., 1987; Houseley and Sinclair, 1998). Further, it has been evidenced, that NMDA receptors present on glutaminergic pathways of the bulbospinal neurones, play an important role in the central processing of the peripheral chemoreceptive information (Dogaš et al., 1995). MK-801, NMDA receptors' blocker injected systemically attenuated the expression of the hypoxic ventilatory effects and reduced peripheral chemoreceptor response to sodium cyanide in the conscious rats (Ohtake et al., 1998).

Administration of NMDA via the peripheral circulation used in the present study affected both components of the breathing pattern, with a predominant effect on the timing component resulting in an apparent impact on the prolongation of the expiratory time. This finding is similar to the post-hypoxic prolongation of expiration in vagotomised rats, which was abolished by the blockade of NMDA receptors in the rat's medulla (Coles et al., 1998). Our report is the first that documents mediation of the respiratory response to systemic NMDA challenge by chemoreceptive pathways. It is assumed to occur via the axonal transport of NMDA receptors in the carotid sinus afferent neurones.

NMDA is considered to have the stimulatory effects on dopamine release from brains of the rat and guinea pig (Kathmann et al., 1999; Whitehead et al., 2001).

It is possible to hold the conviction that NMDA may influence respiration indirectly via another transmitter like dopamine. Carotid body of the rat has been postulated to be mainly the dopaminergic structure (Gonzalez et al., 1995). In fact the response obtained in our study after NMDA injection resembles the decline in tidal volume and frequency of breathing observed by Cardenas and Zapata (1981) after an intravenous dopamine challenge, which relied also on preserved carotid sinus nerves.

The blood pressure response triggered by NMDA: a short-lived rise followed by insignificant decline, occurred independent of the vagal and carotid afferentation and blockade of NMDA receptors. This result warrants that respiratory inhibition evoked by NMDA challenge is not secondary to baroreceptor stimulation. The prompt hypertensive response apparently occurs beyond the baroreceptor afferentation and may depend on an increase in sympathetic drive and /or release of catecholamines induced by NMDA (Ramage and de Daly, 1998). The published evidence on cardiovascular effects of NMDA was focused on the central 
NMDA receptors' activation. However, the data are quite contradictory. It has been shown that NMDA or Lglutamate microdialised to the locus coeruleus or commissural nucleus of the NTS produce large increase in blood pressure (Hall et al., 1998; Marchenko and Sapru, 2000) or contrary to, the depressor response (Merahi et al., 1992; Matsuo et al., 2001). In the sole study converged on the effect of systemic administration of NMDA on the blood pressure, the fourfold lower dose of NMDA used evoked similar to ours, but delayed and not significant effect. This latter was blocked by AP-7 (Sitniewska et al., 2003). The difference might be possibly due to the low dose of the antagonist used in our experiments, insufficient to block the cardiovascular effect of $27 \mu \mathrm{mol} / \mathrm{kg}$ of NMDA. The central origin of the blood pressure reflex could not be ruled out, if NMDA crosses blood-brain barrier. The reflex pathways underlying the effects of NMDA on arterial blood pressure require further experiments.

In conclusion, the results of the present experiments indicate that the carotid chemoreceptive pathway, involving NMDA receptors, mediates the respiratory effects of systemically applied NMDA in rats with disrupted vagal input. The mechanism of triggering the cardiovascular effects by NMDA remains to be elucidated.

\section{Acknowledgements}

Gratitude is expressed to Mrs Teresa Warnawin for her excellent technical assistance.

\section{References}

Aicher, S.A., Sharma, S., Pickel, V.M., 1999. N-methyl-D-aspartate receptors are present in vagal afferents and their dendritic targets in the nucleus tractus solitarius. Neurosci. 91, 119-132.

Berger, I., Gillis, R.A., Vitagliano, S., Panico, W.H., Magee, S., Kelly, M., Norman, W.P., McManigle, J.E., Taveira DaSilva, A.M., 1995. NMDA receptors are involved at the ventrolateral nucleus tractus solitarii for termination of inspiration. Eur. J. Pharmacol. 277, 195-208.

Bonham, A.C., Coles, S.K., McCrimmon, D.R., 1993. Pulmonary stretch receptor afferents activate excitatory amino acid receptors in the nucleus tractus solitarii in rats. J. Physiol. 464, 725-745.

Cardenas, H., Zapata, P., 1981. Dopamine-induced ventilatory depression in the rat, mediated by carotid nerve afferents. Neurosci. Lett. 24 (1), 29-33.
Coles, S., Ernsberger, P., Dick, T.E., 1998. A role of NMDA receptors in posthypoxic frequency decline in the rat. Am. J. Physiol. 43, R1546-R1555.

Dickman, K.G., Youssef, J.G., Said, S.I., 2004. Ionotropic glutamate receptors in lungs and airways molecular basis for glutamate toxicity. Am. J. Respir. Cell Mol. Biol. 30 (2), 139-143.

Dogaš, Z., Stuth, E.A.E., Hopp, F.A., McCrimmon, D.R., Zuperku, E.J., 1995. NMDA receptor- mediated transmission of carotid body chemoreceptor input to expiratory bulbospinal neurones in dogs. J. Physiol. 487 (3), 639-651.

Gonzalez, C., Dinger, B.G., Fidone, S.J., 1995. Mechanisms of carotid body chemoreception. In: Dempsey, J.A., Pack, A.I. (Eds.), Regulation of Breathing, vol. 79. Marcel Dekker, New York, pp. 391-471.

Hall, S., Milne, B., Jhamandas, K., 1998. Excitatory action of $N$ methyl-D-aspartate on the rat locus coeruleus is mediated by nitric oxide: an in vivo voltammetric study. Brain Res. 796 (1/2), 176-186.

Harris, M.B., Milsom, W.K., 2001. The influence of NMDA receptormediated processes on breathing pattern in ground squirrels. Respir. Physiol. 125, 181-197.

Houseley, G.D., Sinclair, J.D., 1998. Localization by kainic acid lesions of neurones transmitting the carotid chemoreceptor stimulus for respiration in rat. J. Physiol. Lond. 406, 99-144.

Kaczyńska, K., Szereda-Przestaszewska, M., 2004. Cardiorespiratory effects of intravenous $N$-methyl-D-Aspartate (NMDA) in anaesthetized rats. Clinic. Exp. Pharmacol. Physiol. 31 (1/2), 101-106.

Kathmann, M., Bauer, U., Schlicker, E., Göthert, M., 1999. Cannabinoid $\mathrm{CB}_{1}$ receptor-mediated inhibition of NMDA- and kainatestimulated noradrenaline and dopamine release in the brain Naunyn-Schmiedeberg's Arch. Pharmacol. 359, 466-470.

Lewis, S.J., Cincotta, M., Verberne, A.J.M., Jarott, B., Lodge, D., Beart, P.M., 1987. Receptor autoradiography with L- $\left[{ }^{3} \mathrm{H}\right]-$ glutamate reveals the presence and axonal transport of glutamate receptors in vagal afferent neurones of the rat. Eur. J. Pharmacol. 144, 413-415.

Lodge, D., Danysz, W., 2000. Introduction to glutamate receptors, their function and pharmacology. In: Lodge, D., Danysz, W., Parsons, C.G. (Eds.), Ionotropic glutamate receptors as therapeutic targets. FP Graham Publishing, Johnson City, Tennessee, pp. 1-27.

Marchenko, V., Sapru, H.N., 2000. Different patterns of respiratory and cardiovascular responses elicited by chemical stimulation of dorsal medulla in the rat. Brain Res. 857, 99-109.

Matsuo, I., Hirooka, Y., Hironaga, K., Eshima, K., Shigematsu, H., Shihara, M., Sakai, K., Takeshita, A., 2001. Glutamate release via NO production evoked by NMDA in the NTS enhances hypotension and bradycardia in vivo. Am. J. Physiol. Regul. Integr. Comp. Physiol. 280, 1285-1291.

McCrimmon, D.R., Mitchell, G.S., Dekin, M.S., 1995. Glutamate, GABA, and serotonin in ventilatory control. In: Dempsey, J.A., Pack, A.I. (Eds.), Regulation of breathing. M. Dekker, New York, Basel, Hong Kong, pp. 151-218.

Merahi, N., Orer, H.S., Laguzzi, R., 1992. 5- $\mathrm{HT}_{2}$ receptors in the nucleus tractus solitarius: characterisation and role in cardiovascular regulation in the rat. Brain Res. 575, 74-78. 
Ohtake, P.J., Torres, J.E., Gozal, Y.M., Graff, G.R., Gozal, D., 1998. NMDA receptors mediate peripheral chemoreceptor afferent input in the conscious rat. J. Appl. Physiol. 84 (3), 853-861.

Okada, J., Miura, M., 1992. Transmitter substances contained in the petrosal ganglion cells determined by a double-labeled method in the rat. Neurosci. Lett. 146, 33-36.

Ramage, A.G., de Daly, M.B., 1998. The central action of 5- $\mathrm{HT}_{2}$ receptor agonist 1-\{2 5-dimethoxy-4-iodophenyl $\}-2-$ amionopropane (DOI) on cardiac inotropy and vascular resistance in the anaesthetized cat. Br. J. Pharmacol. 125, 1172-1179.

Sapru, H.N., Krieger, A.J., 1977. Carotid and aortic chemoreceptor function in the rat. J. Appl. Physiol. 42, 344-348

Shigemoto, R., Ohishi, H., Nakanishi, S., Mizuno, N., 1992. Expression of the mRNA for the rat NMDA receptor (NMDAR1) in the sensory and autonomic ganglion neurons. Neurosci. Lett. 144, 229-232.

Sitniewska, E.M., Wiśniewska, R.J., Wiśniewski, K. (2003). The role of ionotropic receptors of glutaminic acid in cardiovascular system. Amino Acids DOI: 10.1007/s00726-002-0342-4.

Torrealba, F., Bustos, G., Montero, V.M., 1996. Glutamate in the glomus cells of the cat carotid body: immunocytochemistry and in vitro release. Neurochem. Int. 28, 625-631.

Whitehead, K.J., Rose, S., Jenner, P., 2001. Involvement of intrinsic cholinergic and GABAergic innervation in the effect of NMDA on striatal dopamine efflux and metabolism as assessed by microdialysis studies in freely moving rats. Eur. J. Neurosci. 14 (5), 850-851. 